\title{
A predicted protein interactome for rice
}

\author{
Chai-Ling Ho ${ }^{1}$, Yingzhou Wu ${ }^{2}$, Hong-bin Shen ${ }^{3}$, Nicholas J Provart ${ }^{4}$ and Matt Geisler ${ }^{5^{*}}$
}

\begin{abstract}
Background: Protein-protein interactions (PPIs) create the steps in signaling and regulatory networks central to most fundamental biological processes. It is possible to predict these interactions by making use of experimentally determined orthologous interactions in other species.

Results: In this study, prediction of PPIs in rice was carried out by the interolog method of mapping deduced orthologous genes to protein interactions supported by experimental evidence from reference organisms. We predicted 37112 interactions for 4567 rice proteins, including 1671 predicted self interactions (homo-interactions) and 35441 predicted interactions between different proteins (hetero-interactions). These matched 168 of 675 experimentally-determined interactions in rice. Interacting proteins were significantly more co-expressed than expected by chance, which is typical of experimentally-determined interactomes. The rice interacting proteins were divided topologically into 981 free ends (proteins with single interactions), 499 pipes (proteins with two interactions) and 3087 hubs of different sizes ranging from three to more than 100 interactions.
\end{abstract}

Conclusions: This predicted rice interactome extends known pathways and improves functional annotation of unknown rice proteins and networks in rice, and is easily explored with software tools presented here.

\section{Background}

Protein-protein interactions (PPIs) are essential for many fundamental biological processes. With the advent of high-throughput approaches, genome-wide networks of PPIs have been generated in Saccharomyces cerevisiae (Uetz et al., 2000; Miller et al., 2005; Gandhi et al., 2006), Drosophila melanogaster (Giot et al., 2003), Caenorhabditis elegans (Li et al., 2004), Homo sapiens (Rual et al., 2005) and other organisms. Recently, a large scale map of 6200 PPIs was completed for Arabidopsis (Arabidopsis interactome mapping consortium, 2011). Another medium-scale yeast two-hybrid screen on proteins involved in the two-component signaling pathway of Arabdidopsis thaliana has revealed 160 interactions of which 136 were novel (Dortay et al., 2008). Networks of rice genes associated with stress response, seed development and cell cycle mediated by cyclin were built from the results generated from yeast two hybrids (Cooper et al., 2003a,b). In addition, a rice kinase-protein interaction map of 116 representative rice kinases and their

\footnotetext{
* Correspondence: mgeisler@plant.siu.edu

${ }^{5}$ Department of Plant Biology, Southern Illinois University Carbondale, 1125 Lincoln Ave., Life Science II, Carbondale, IL 62901-6509, USA Full list of author information is available at the end of the article
}

interacting proteins was generated from the results of yeast two hybrids (Ding et al., 2009).

Prediction of PPIs is made possible in organisms lacking experimental determination of PPIs using the PPI networks established in reference organisms. In this approach, orthologous genes are deduced using prediction algorithms and mapped to protein interactions supported by experimental evidence from reference organisms retrieved from publicly available databases such as Biomolecular Interaction Network Database (BIND; Bader et al., 2001), Molecular Interaction Database (MINT; Zanzoni et al., 2002; Ceol et al., 2009), Munich Information Center for Protein Sequences (MIPS; Pagel et al., 2005), Database of Interacting Proteins (DIP; Salwinski et al., 2004), IntAct (http://www.ebi.ac.uk/intact; Aranda et al., 2010) and Biological General Repository for Interaction Data sets (BioGRID; Breitkreutz et al., 2008). Using this approach, a predicted interactome of Arabidopsis thaliana was made consisting 1159 high confidence, 5913 medium confidence and 12907 low confidence interactions. This was established using a confidence scoring based method on the number of different data sets in which the interaction was recorded, the number of different types of experiments supported the interactions, and the number of species in which the interaction was discovered (Geisler-Lee et al.,

\section{它}

(c) 2012 Ho et al.; licensee Springer. This is an Open Access article distributed under the terms of the Creative Commons Attribution License (http://creativecommons.org/licenses/by/2.0), which permits unrestricted use, distribution, and reproduction in any medium, provided the original work is properly cited. 
2007). In addition, the data on subcellular localization and co-expression of interacting proteins were integrated into the deduction of PPIs to strengthen the confidence of the resulting predicted interactome. The predicted interactome in Arabidopsis revealed that many of the most highly conserved proteins were also the most highly connected hubs involved in important signaling complexes, and revealed the preservation of original functions of nuclear-located pathways in non-photosynthetic reference organisms in the chloroplasts of higher plants post endosymbiosis (Geisler-Lee et al., 2007). The Arabidopsis predicted interactome has enabled researchers to fruitfully generate and test network and protein interaction hypotheses (e.g. Liu and Howell 2010, Gu et al. 2008).

In this study, a similar approach was used to predict the interactome of rice with the aim to expand the current understanding of PPIs in monocot based on our predicted interactome. A second goal is to provide a tool that leads to useful hypothesis generation.

\section{Results and discussion}

Predicted rice interactions

In this study, a rice protein-protein interaction network was predicted based on the universality of conserved protein function among different organisms. This was undertaken with the assumption that evolutionarily conserved orthologous proteins are likely to retain their interactions with other similarly conserved proteins. Using ortholog prediction algorithm, 13070 rice genes (23\% of rice genome) had an ortholog that matched at least one of the eleven reference organisms (Arabidopsis thaliana, Homo sapiens, Mus musculus, Rattus norvegicus, Drosophila melanogaster, Caenorhabditis elegans, Saccharomyces cerevisiae, Schizosaccharomyces pombe, Escherichia coli, Bacillus subtilis, Helicobacter pylori). A confidence value ( $\mathrm{CV}$, see Methods) was calculated to estimate the strength of experimental support for each predicted interaction. We have identified 37112 predicted interactions for 4567 rice proteins (Additional file 1: Table S1), whereby 1671 are predicted self interactions and 35441 are interactions between different proteins. These interactions were classified into 2904 interactions with high confidence $(\mathrm{CV}>10), 11152$ interactions with medium confidence (CV between 2 and 10) and 23056 interactions with low confidence $(\mathrm{CV}=1)$ (Figure 1). Table 1 lists the twenty protein interactions with the highest confidence, owing to repeatability using different experimental techniques and species. Among these interactions are DNA repair pathways involving RAD50, 51, 54, MCM2, 5, 6, UVH1, MRE11, and others; Cell cycle control pathways with $\mathrm{CDC} 2$, cyclin dependent kinases, SNF1-related protein kinase; and the transcription initiation complex. While many of these interactions have been well studied in human and yeast, equivalent studies are lacking for most of these in rice. All genes in Table 1 have been functionally annotated in rice only by sequence homology, often only to the level of general gene family and not their specific role. By adding predicted interactions, the exact role for each protein in the network can be more specifically hypothesized.

\section{Conservation in interactions}

The same protein interactions were found in many species, despite the incompleteness of the experimentallydetermined interactomes (Wiles et al. 2010). The same pathway interactions for DNA repair is found in 5 other eukaryotic species, including the pathway components RAD51, DNA replication licensing factors MCM2,5,6 and PROLIFERA; DNA polymerase subunits and actinlike proteins (Table 2). These are also interactions with the highest confidence (with CV more than 400). We identified 793 interactions that were present in at least two different species and orthologous genes were found in rice. Proteins in rice with highest connectivity (number of different interactions) were not always the most evolutionarily conserved, which differs from the predicted Arabidopsis interactome (Geisler-Lee et al., 2007). Three of the most conserved protein interactions in rice between DNA polymerase catalytic subunit and DNA polymerase subunit $\mathrm{B}$, small nuclear ribonucleoproteins SmD2 and F and self-interaction of UTP-glucose-1phosphate uridyltransferase were also among the most conserved in Arabidopsis (Geisler-Lee et al., 2007). The other highly conserved interactions between the two species are different (Table 2). More rice orthologs $(13,070)$ were identified in comparison to Arabidopsis $(10,776)$ possibly owing to a larger genome in rice, and there was an increased pool of reference species.

\section{Experimental verification of predicted rice interactions}

A gold standard of 675 experimentally confirmed interactions was abstracted from IntAct (http://www.ebi.ac. uk/intact/; Additional file 2: Table S2). Of these 675 experimentally-determined interactions, there were 168 interactions that were also predicted by our method. This is a very high overlap, with a significance p-value of less than $10^{-99}$ when compared to a chance overlap with an equal number of random protein pairs. This is especially significant when considering not all experimentally-determined interactions were also evolutionarily conserved, and thus not easily detectable by interacting orthologs. A PubMed ID was assigned to each of the gold standard interactions in our data sets. The remaining experimentally-determined interactions were loaded into the Rice Interactions Viewer (RIV) database. 


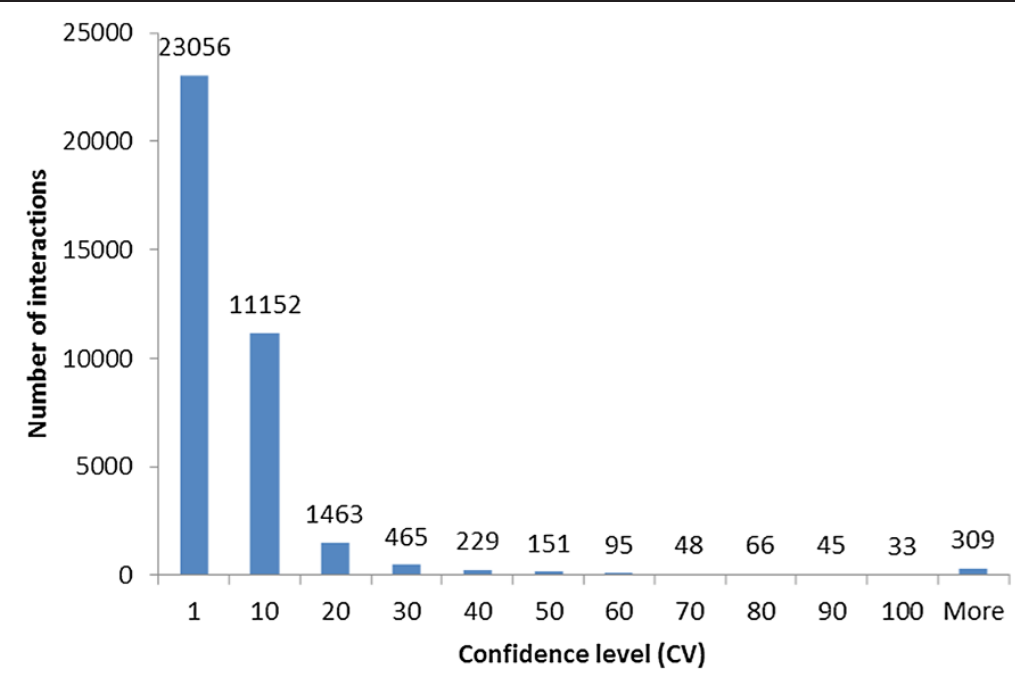

Figure 1 Confidence Values of predicted rice protein-protein interactions. Confidence value was calculated from the overall supporting evidence with a multiplier for interactions found by different experimental methodologies and found in different reference species. Most predicted interactions were of low confidence (1), but 11152 interactions had medium and 2904 had high confidence levels. 37112 unique interactions involving 4567 proteins were predicted which included 1671 self interactions and 35441 hetero-interactions.

Table 1 Twenty rice protein interactions with the highest confidence

\begin{tabular}{|c|c|c|c|c|}
\hline Locus $A^{*}$ & Locus B* & Protein A & Protein B & CV \\
\hline Os11g40150 & Os11g40150 & DNA repair protein RAD51 homolog A & DNA repair protein RAD51 homolog A & 2080 \\
\hline Os03g53960 & Os10g28040 & transcriptional adaptor & $\begin{array}{l}\text { general control of amino acid synthesis protein } \\
\text { 5-like } 2\end{array}$ & 2048 \\
\hline Os01g72880 & Os02g37920 & MUTL protein homolog 1 & ATP binding protein & 2040 \\
\hline Os02g30800 & Os05g06840 & $\begin{array}{l}\text { DNA polymerase family B, exonuclease domain containing } \\
\text { protein, expressed }\end{array}$ & DNA polymerase epsilon subunit 2 & 1404 \\
\hline Os07g02350 & Os10g41520 & casein kinase II subunit alpha-2 & casein kinase II subunit beta- 4 & 1368 \\
\hline Os03g63940 & Os05g45420 & protein kinase AKINbetagamma-2 & $\begin{array}{l}\text { SNF1-related protein kinase catalytic alpha } \\
\text { subunit KIN10 }\end{array}$ & 1281 \\
\hline Os02g29464 & Os04g54340 & DNA repair protein RAD50 & double-strand break repair protein MRE11 & 1254 \\
\hline Os02g38340 & Os08g28190 & actin-like protein 3 & actin-like protein 2 & 1155 \\
\hline Os04g43300 & Os05g43610 & ATBRCA1 & ATBRCA1 & 1040 \\
\hline Os06g43790 & Os12g39070 & HAF01 & TATA-binding protein 2 & 1026 \\
\hline Os02g52510 & Os11g40150 & DNA repair protein RAD54-like, putative & DNA repair protein RAD51 homolog A & 952 \\
\hline Os03g46650 & Os05g26890 & guanine nucleotide-binding protein beta subunit & $\begin{array}{l}\text { guanine nucleotide-binding protein alpha-1 } \\
\text { subunit }\end{array}$ & 936 \\
\hline Os11g29380 & Os12g37400 & DNA replication licensing factor $\mathrm{Mcm} 2$ & PROLIFERA protein & 800 \\
\hline Os03g01100 & Os10g37490 & DNA repair endonuclease UVH1 & mating-type switching protein swi10 & 783 \\
\hline Os01g08540 & Os05g19270 & DNA mismatch repair protein MSH6-2 & DNA mismatch repair protein $\mathrm{MSH} 2$ & 774 \\
\hline Os01g32750 & Os03g29470 & TBP-associated $59 \mathrm{kDa}$ subunit protein & transcription initiation factor TFIID subunit 9B & 756 \\
\hline Os01g64820 & Os12g13950 & DNA polymerase alpha catalytic subunit & DNA polymerase alpha subunit B & 720 \\
\hline Os01g07110 & Os11g40150 & BRCA2 repeat family protein & DNA repair protein RAD51 homolog A & 714 \\
\hline Os06g08770 & Os07g08170 & ruvB-like 2 & ruvB-like 1 & 651 \\
\hline Os03g02680 & Os03g05300 & cell division control protein 2 homolog 1 & cyclin-dependent kinases regulatory subunit & 648 \\
\hline
\end{tabular}

*rice locus identifiers are also written as e.g. LOC_Os11g40150. 
Table 2 Twenty most conserved rice protein interactions

\begin{tabular}{|c|c|c|c|c|c|}
\hline Locus $A^{*}$ & Locus B* & Protein A & Protein B & Species & CV \\
\hline Os11g40150 & Os11g40150 & DNA repair protein RAD51 homolog A & DNA repair protein RAD51 homolog A & 5 & 2080 \\
\hline Os02g55410 & Os11g29380 & DNA replication licensing factor MCM5 & DNA replication licensing factor $\mathrm{Mcm} 2$ & 5 & 560 \\
\hline Os01g64820 & Os12g13950 & DNA polymerase alpha catalytic subunit & DNA polymerase alpha subunit B & 5 & 720 \\
\hline Os02g38340 & Os08g28190 & actin-like protein 3 & actin-like protein 2 & 5 & 1155 \\
\hline Os07g22400 & Os12g13950 & DNA primase large subunit & DNA polymerase alpha subunit B & 5 & 450 \\
\hline Os01g36390 & Os11g29380 & DNA replication licensing factor $\mathrm{mcm} 4$ & DNA replication licensing factor $\mathrm{Mcm} 2$ & 5 & 420 \\
\hline Os11g29380 & Os12g37400 & DNA replication licensing factor $\mathrm{Mcm} 2$ & PROLIFERA protein & 5 & 800 \\
\hline Os05g14590 & Os11g29380 & DNA replication licensing factor MCM6 & DNA replication licensing factor $\mathrm{Mcm} 2$ & 5 & 595 \\
\hline Os01g72880 & Os02g37920 & MUTL protein homolog 1 & ATP binding protein & 4 & 2040 \\
\hline Os09g38030 & Os09g38030 & UTP-glucose-1-phosphate uridylyltransferase & UTP-glucose-1-phosphate uridylyltransferase & 4 & 176 \\
\hline Os05g24970 & Os11g43620 & small nuclear ribonucleoprotein Sm D2 & small nuclear ribonucleoprotein $\mathrm{F}$ & 4 & 272 \\
\hline Os01g71990 & Os01g71990 & pyrroline-5-carboxylate reductase & pyrroline-5-carboxylate reductase & 4 & 144 \\
\hline Os05g39850 & Os11g29380 & DNA replication licensing factor MCM3 & DNA replication licensing factor $\mathrm{Mcm} 2$ & 4 & 476 \\
\hline Os02g55410 & Os05g39850 & DNA replication licensing factor MCM5 & DNA replication licensing factor MCM3 & 4 & 448 \\
\hline Os07g02350 & Os10g41520 & casein kinase II subunit alpha-2 & casein kinase II subunit beta- 4 & 4 & 1368 \\
\hline Os03g53960 & Os10g28040 & transcriptional adaptor & general control of amino acid synthesis protein 5-like 2 & 4 & 2048 \\
\hline Os06g43790 & Os06g44030 & HAF01 & transcription initiation factor TFIID subunit 5 & 4 & 580 \\
\hline Os02g52510 & Os11g40150 & DNA repair protein RAD54-like & DNA repair protein RAD51 homolog A & 4 & 952 \\
\hline Os01g53600 & Os09g33930 & farnesyltransferase beta subunit & farnesyltransferase/geranylgeranyltransferase type IA & 4 & 456 \\
\hline Os05g32310 & Os08g08040 & SAD1 & LSM7 homolog, U6 small nuclear RNA associated & 4 & 224 \\
\hline
\end{tabular}

*rice locus identifiers are also written as e.g. LOC_Os11g40150.

\section{Interactome topology}

The rice interacting proteins were divided into free ends (981 proteins with single interactions), pipes (499 proteins with two interactions) and hubs of different sizes ranging from three to more than 100 interactions. The largest class of proteins is that of medium hubs (between 11 to 50 interactions). The interacting proteins had an average of 14-15 partners, which is comparable to Drosophila (9 interacting partners) and Arabidopsis (11 interacting partners) and smaller than in yeast (22 interacting partners). Table 3 lists the most highly connected rice protein interaction hubs, including heat shock protein 81-1, elongation factor 1-alpha, vacuolar ATP synthase subunit $\mathrm{B}$ isoform 1. Among these proteins, ubiquitinconjugating enzyme E2, ubiquitin-like protein SMT3, fibrallarin-2 were also found among the twenty most highly connected Arabidopsis interaction hubs (GeislerLee et al., 2007).

\section{Protein domain enrichment in rice interologs}

Protein-Protein interactions are often mediated by protein interaction domains (PIDs) which bind to other domains (domain-domain interactions), or short conserved amino acid motifs in the partner protein. PIDs that interact most frequently among the rice protein pairs (interologs) were determined using annotation by PFAM domain models (see methods). In rice, the most
Table 3 Twenty most highly connected rice protein interaction hubs

\begin{tabular}{lll}
\hline Locus & Protein Description & Edges \\
\hline Os08g39140 & heat shock protein 81-1 & 686 \\
Os03g08050 & elongation factor 1-alpha & 410 \\
Os06g37180 & vacuolar ATP synthase subunit B isoform 1 & 298 \\
Os01g38970 & carbamoyl-phosphate synthase large chain & 276 \\
Os07g08330 & 60 S ribosomal protein L4 & 272 \\
Os01g62840 & mannose-1-phosphate guanyltransferase & 245 \\
Os01g73310 & actin-1 & 234 \\
Os01g62244 & ubiquitin-conjugating enzyme E2 7 & 224 \\
Os10g32550 & chaperonin CPN60-1, mitochondrial precursor & 214 \\
Os11g04070 & 60 S acidic ribosomal protein P0 & 197 \\
Os07g31370 & ras-related protein Rab-6A & 187 \\
Os07g43360 & histone acetyltransferase MYST1 & 182 \\
Os05g38530 & heat shock cognate 70 kDa protein & 180 \\
Os03g45920 & tubulin beta-8 chain & 170 \\
Os09g38020 & histone H4 & 169 \\
Os06g38470 & histone deacetylase & 168 \\
Os11g14220 & tubulin alpha-3 chain & 167 \\
Os01g68940 & ubiquitin-like protein SMT3 & 167 \\
Os05g08360 & fibrillarin-2 & 162 \\
Os03g13970 & 26 S proteasome non-ATPase regulatory subunit 4 & 161 \\
\hline
\end{tabular}


enriched domains among the interactors included known interaction domains PF00400 (WD40), PF00069 (protein kinase), PF00270 (DEAD), and PF02985 (HEAT). Looking at domain-domain interactions amongst interacting pairs, domain pairs (with one domain in each interacting protein) with extremely high fold of enrichment (vs. random pairing) were often otherwise rare domains among the protein interactions. Among the twenty most frequent PFAM interacting pairs were 8 self interactions between homodomains and 12 interactions between different PFAM domains. Of these $14(70 \%)$ were enriched more than 2 fold, with the highest fold of enrichment (134 fold) for interaction between the homodomains of the proteasome (PF00227; Table 4). The proteasome is a proteinase complex involved in an ATP/ubiquitin-dependent nonlysosomal proteolytic pathway which liberates the cell of misfolded or damaged proteins and controls the level of certain regulatory proteins (Goldberg and Rock, 1992; Hilt and Wolf, 1996; Rivett, 1993; Wilk, 1993). Since its function is crucial and is composed of about 28 distinct subunits that form a highly ordered structure in eukaryotes, it is not surprising that this PFAM domain shows the highest fold of enrichment in the predicted rice interactions. These interactions form a distinct subnetwork of highly conserved interactions.

\section{Predicted rice interactome subnetworks}

In this study, we present a predicted interactome from rice that is useful for hypothesis generation towards better understanding of protein-protein interactions in rice and also other monocotyledonous plants. We have constructed a predicted rice MADS network consisting of 19 rice MADS-box family members which was determined partially by experiments in rice (Figure 2) which has been expanded using interologs from Arabidopsis, (de Folter et al. 2005) indicated by blue lines in Figure 2. Although these genes are plant specific, the degree of interconnectivity among MADS box genes is not surprising as many proteins form functional complexes consisting of homodimers or heterodimers (Pelaz et al., 2000; Theissen and Melzer, 2007) that have diverse roles. In addition, predicted interactions have connected several rice MADS proteins to proteins that do not belong to the MADS-box family, e.g. LEUNIG (LUG), SEUSS (SEU), and PROLIFERA and more than 10 other proteins involved in diverse functions including DNA metabolism (RuvB-like proteins; Gorynia et al., 2006), initiation of transcription TATA binding protein protein associated factors (TAFs) and a general transcription factor, TFIID (Cler et al., 2009), cell cycle progression (mannose-1-phosphate guanyltransferase, Donoso et al., 2005), and cell division (cell division

Table 4 Twenty most frequent domain pairs between interacting partners

\begin{tabular}{|c|c|c|c|}
\hline Domain IDs in interacting pairs & Domain names & Number of interactions observed & Fold enrichment vs. random \\
\hline PF00400 PF00400 & WD40 : WD40 & 166 & 2.4280 \\
\hline PF00271 PF00270 & Helicase C : DEAD & 131 & 8.0228 \\
\hline PF00069 PF00069 & Pkinase : Pkinase & 120 & 0.0383 \\
\hline PF00227 PF00227 & Proteasome : Proteasome & 114 & 133.8151 \\
\hline PF00076 PF00076 & rrm :rrm & 112 & 1.2412 \\
\hline PF01486 PF00319 & K-box : SRF-TF & 104 & 30.0497 \\
\hline PF00271 PF00271 & Helicase_C : Helicase_C & 101 & 3.5858 \\
\hline PF00400 PF00271 & WD40 : Helicase_C & 99 & 2.2560 \\
\hline PF00271 PF00076 & Helicase C: rrm & 94 & 1.8646 \\
\hline PF01423 PF01423 & $\mathrm{Sm}: \mathrm{Sm}$ & 91 & 116.3077 \\
\hline PF07714 PF00069 & Pkinase_Tyr : Pkinase & 91 & 0.0473 \\
\hline PF00270 PF00076 & DEAD : rrm & 74 & 2.5320 \\
\hline PF00400 PF00069 & WD40 : Pkinase & 70 & 0.1512 \\
\hline PF00400 PF00076 & WD40 : rrm & 70 & 0.8912 \\
\hline PF00069 PF00036 & Pkinase : efhand & 70 & 0.1994 \\
\hline PF02985 PF00400 & HEAT : WD40 & 65 & 2.5236 \\
\hline PF00400 PF00118 & WD40 : cpn60_TCP1 & 62 & 8.8624 \\
\hline PF00004 PF00004 & AAA : AAA & 60 & 3.4780 \\
\hline PF00400 PF00270 & WD40 : DEAD & 59 & 2.3192 \\
\hline PF00270 PF00270 & DEAD : DEAD & 58 & 6.1273 \\
\hline
\end{tabular}




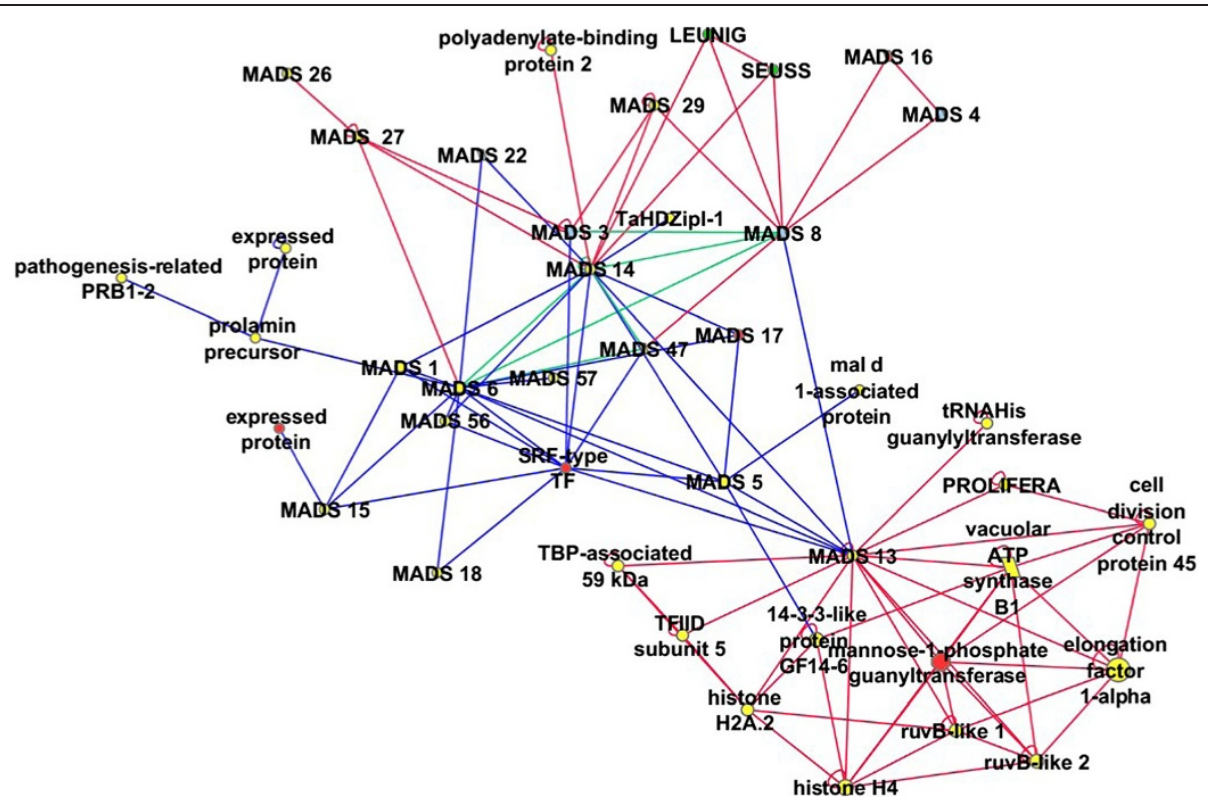

Figure 2 MADS network in rice expanded by predicted interactions. The core interactions between rice MADS box proteins were experimentally determined by de Folter et al. 2005 (Circles: rice proteins, Blue edges: experimentally determined interactions). This network is expanded by predicted interactions (Red edges), and several experimentally determined interactions were also predicted (Green edges).

co-repressors that form a co-repressor complex with MADS box dimers, APETALA1 (AP1) AP1- AGAMOUSLIKE 24 (AGL24) and AP1-SHORT VEGETATIVE PHASE (SVP) to repress AGAMOUS (AG) gene in flowers (Gregis et al., 2006). SEU has been demonstrated to interact with AP1 and SEPALLATA3 (SEP3) to bridge the interaction between AP1/SEP3 and LUG in Arabidopsis (Sridhar et al., 2006) resulting in transcription repression during flower development. OsMADS14 and OsMADS8 which are connected to SEU here, could be the rice orthologs for AP1 and SEP, while OsMADS13 could be a rice ortholog for AGL11 (Arora et al., 2007) which was reported to be preferentially expressed in ovule (Rounsley et al. 1995) and carpel (Yung et al., 1999). OsMADS13 was also connected to PROLIFERA (PRL) which encodes a DNA replication licensing factor Mcm7. In Arabidopsis, $P R L$ was demonstrated to be expressed in dividing cells in the palisade layer of the leaf, founder cells of initiating flower primordial, and central cell nucleus of mature mega gametophytes (Springer et al., 2000) whereas PROLIFERA protein was reported during $\mathrm{G}(1)$ phase of the cell cycle. The interaction network involving PROLIFERA was further expanded through interologs in other eukaryotes (Figure 3a), showing the relationship between the MADS box network to conserved network of cell division and cell cycle regulators.

The predicted rice interactome is also useful for the functional annotation of expressed proteins with unknown functions through their associations with known proteins, or known pathways in the predicted network.
Subnetworks of interacting proteins were constructed around MAP kinase signaling proteins identified by homology (Figure $3 \mathrm{~b}$ ) and the known protein phosphatase OSPP2C4, (Figure 3c). These subnetworks contained many expected interactions but also included both metabolic enzymes (e.g. acyl co-enzyme A synthase in Figure $3 \mathrm{c}$ ) and regulators (e.g. serine/threonine phosphatase $2 \mathrm{~A}$ regulatory subunits), as well as unexpected connections between metabolic enzymes, signaling and ribosomal proteins.

\section{Coexpression of interologs}

Proteins which physically interact are often expressed at the same time in the same tissue (Narayanan et al. 2010). This is possibly due to common transcription factors controlling individual members of a protein complex or pathway, a common network motif known as a single input module (Alon, 2007). Especially in metabolism, co-expressed enzymes utilize "just in time" mechanisms of regulation so that they do not waste energy producing idle proteins (Zaslaver et al., 2004). Thus mRNA levels of the transcripts for interacting proteins also frequently co-vary, as do levels of transcripts for proteins that are part of a signaling chain or enzymes in a metabolic pathway that is coordinately stimulated or suppressed. A network of gene co-expression can itself be informative in pathway reconstruction. As a result, if a pair of proteins is positively coexpressed, the confidence level to predict the pair interacts is generally high (Geisler-Lee et al. 2007). The co-expression level of each 


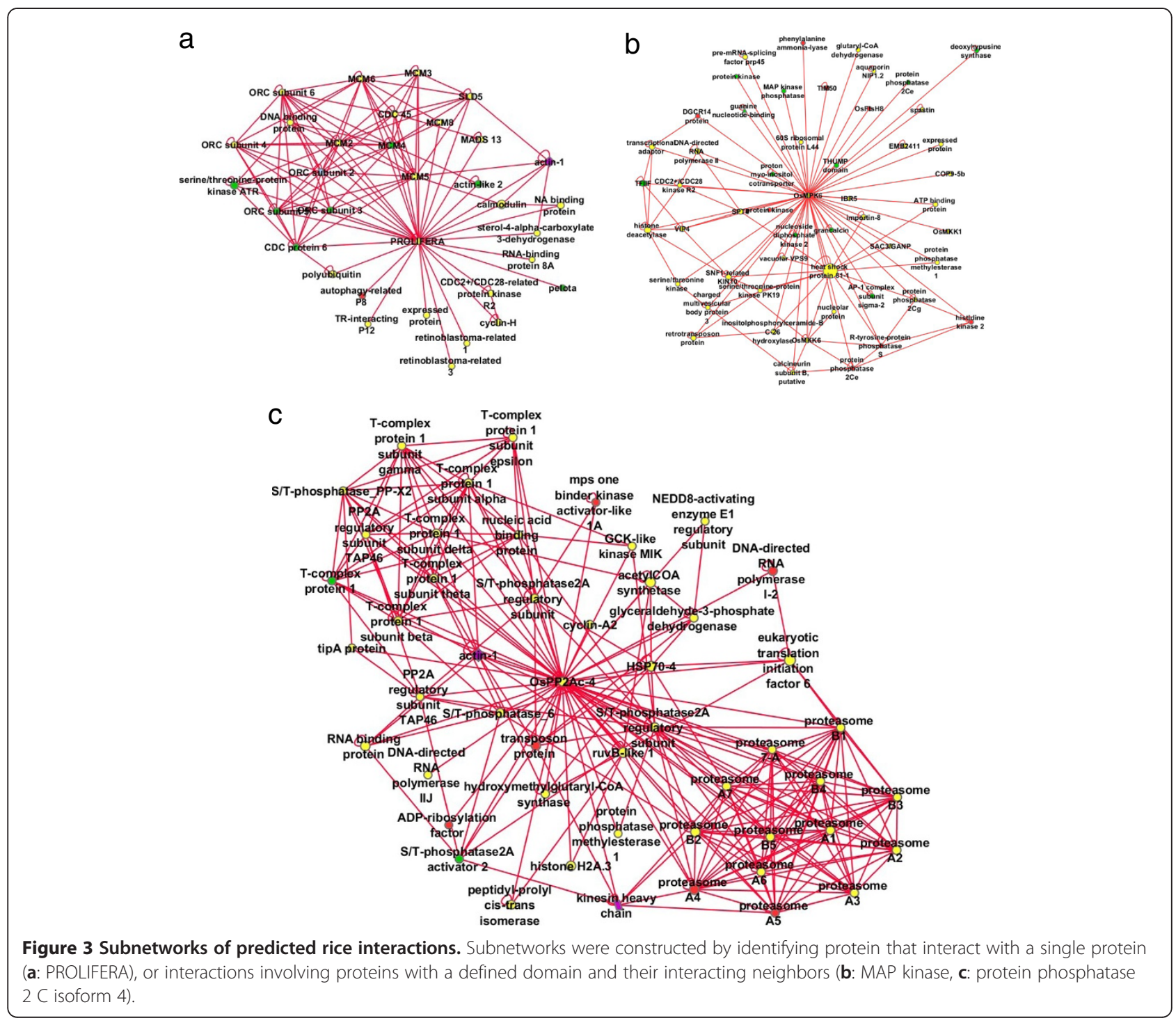

interolog is measured by Pearson Correlation Coefficient (PCC) which is computed from available rice Affymetrix microarray data which includes 165 data sets in total from diverse tissues and treatments (Additional file 3: Table S3). In comparison to the randomly generated gene pairs (see methods), our predicted interologs exhibited a strong and statistically significant trend of coexpression (Figure 4). Predicted interologs with low coexpression were also found in the analysis. Not all proteins with physical interactions are co-expressed, as may be the case with rate limiting steps or proteins regulated at the post-transcriptional level. One protein can be constitutively expressed while the other interacting partner is expressed under certain conditions only, especially where a pathway branches into two downstream paths. Protein relocation to another compartment or even another tissue is also a possible cause. Collectively, these are called "limiting factor" based regulation mechanisms. Thus lack of expression correlation does not necessarily imply that proteins do not interact. Indeed the combination of co-expression and predicted physical interaction reveals the regulation mechanisms involved in that pathway as either "just in time", or "limiting factor" based. Overall, however, a positive correlation was found between the interolog coexpression (PCC) and the interolog confidence level $(\mathrm{CV})$, indicating that "just in time" regulation is more common.

\section{Subcellular localization of interologs}

In a general sense, for a pair of proteins to physically interact, each protein must be located at same or adjacent subcellular compartments either permanently or transiently. Thus it is foreseeable that interacting proteins are more likely to have the same or adjacent subcellular localization. We have performed subcellular 

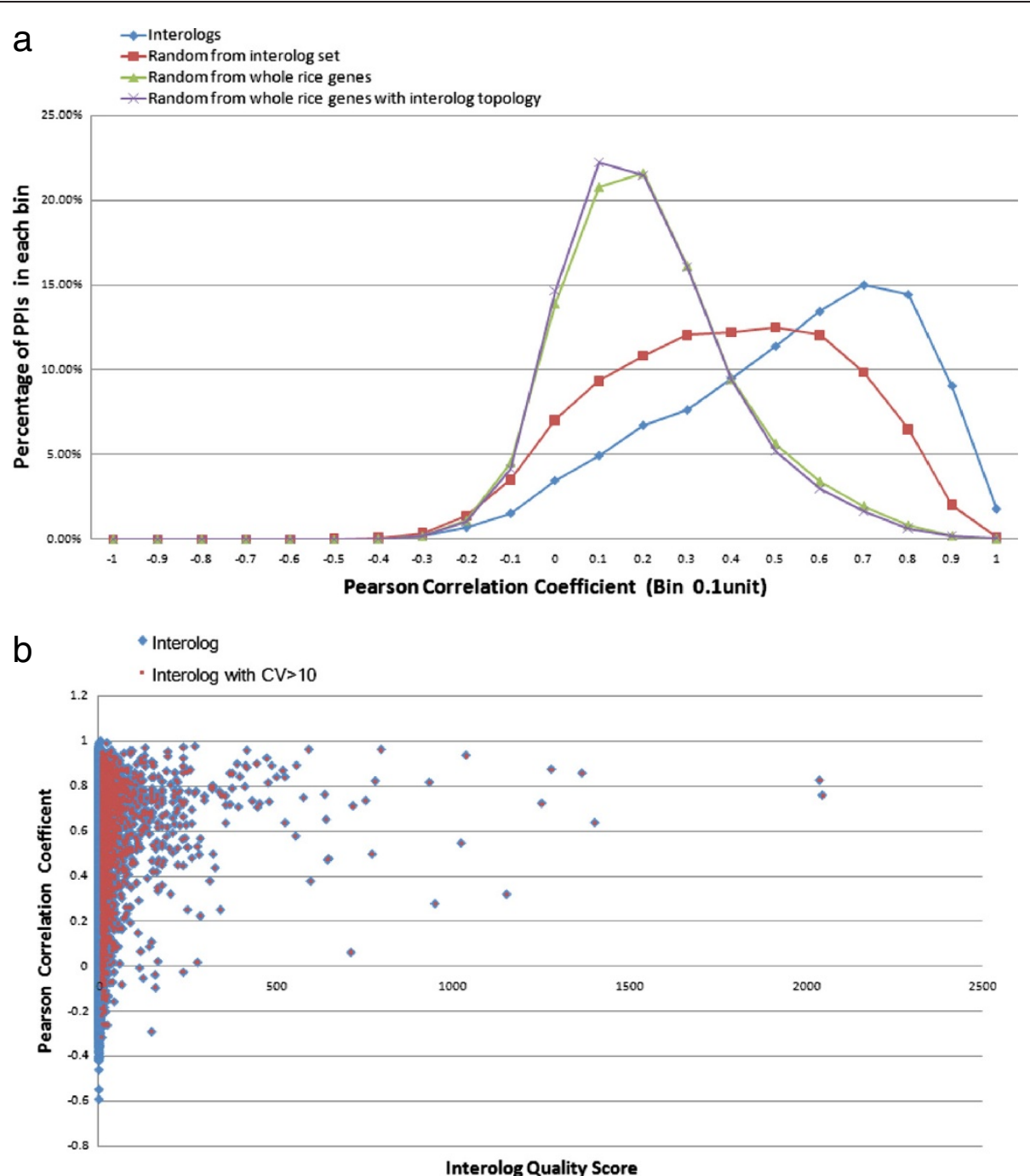

Figure 4 Co-expression of interologs. a, The PCC for 37,112 predicted interaction pairs was calculated and plotted as the number of pairs in each Pearson correlation coefficient range (blue curve). The PCC calculation is also performed for 37,112 randomly selected pairs of rice genes from within our interactome (red curve), from the whole rice genes (green curve), or from whole rice genes such that the topology of the random network was the as that of our predicted interactome in terms of node degree distribution (purple curve). $\mathbf{b}$, The interolog CV was plotted against the PCC for each pair (blue dots). The red dots are the interologs whose CV is larger than 10.

localization enrichment analysis to validate this trend. Unlike the similar approach for Arabidopsis where extensive subcellular localization information can be retrieved from SUBA (Geisler-Lee et al. 2007), comprehensive experimentally derived subcellular localization data for rice are not available. Fortunately, many computational methods are available for predicting protein subcellular localization. Some machine learning-based methods that use sequence-derived features to predict localization have reached remarkable levels of performance. Therefore, we adopted one of these computational methods named Plant-mPloc (http://www.csbio.sjtu.edu. cn/bioinf/plant-multi/; Chou and Shen, 2010) to predict the subcellular localization of each protein in our interactome. Since Plant-mPloc is able to cover 12 distinct compartments, 78 distinct compartment pairs were used for analysis. Figure 5 shows the observed number of interologs of each possible subcellular compartment pairs. $\mathrm{P}$ values associated with the deviation of these numbers from a random interactome network with the same properties (see "Material and Methods") were used to evaluate the statistical significance of enrichment or depletion. Most of the statistically significant enriched compartment pairs are those paired with same compartment or adjacent compartments such as ER and Golgi (Figure 5). A significant number of cytosolic localized proteins are predicted to interact with nuclear and mitochondrial localized proteins, indicating possible import/ 


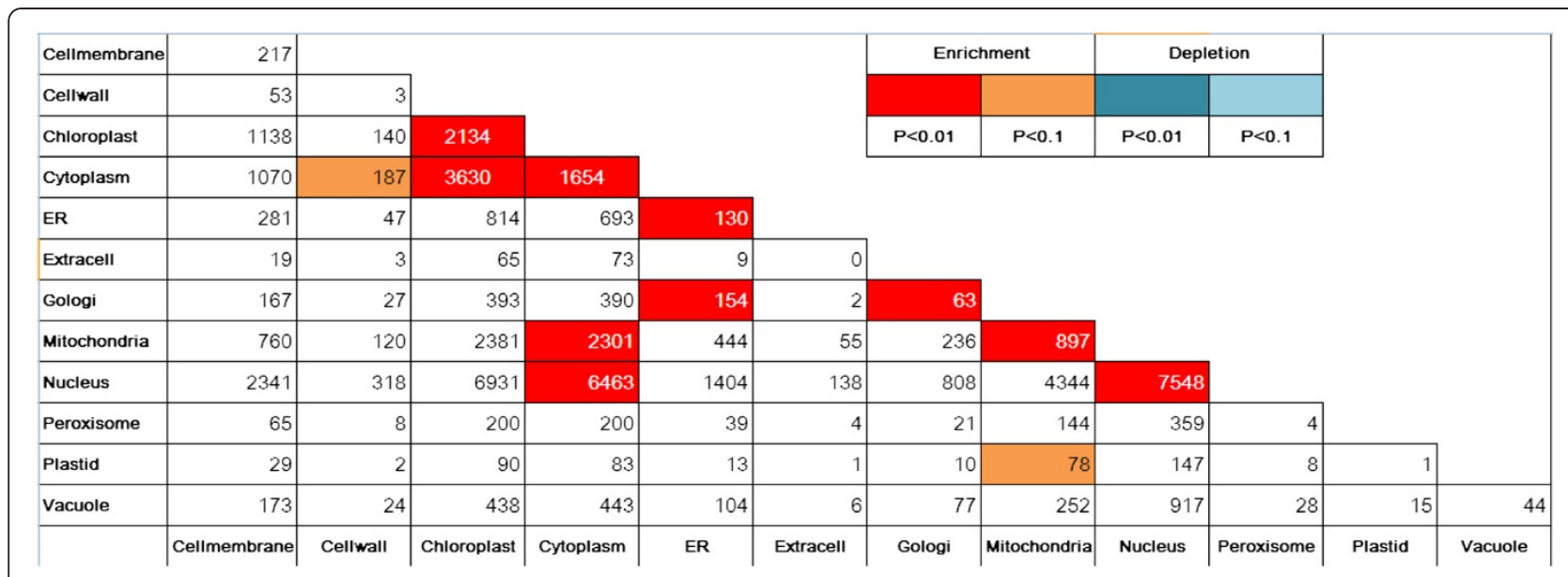

Figure 5 Co-localization of interologs. Analysis of all interaction protein pairs in which both partners were assigned to a subcellular compartment. Compartment pairs that showed enriched or depleted numbers of interactions (compared to chance) are color coded.

export regulation and dual localized proteins such as transcription factors. No statistically significant depletion was found in this analysis. The results differ slightly from what we observed in the case of the predicted interactome for Arabidopsis (Geisler-Lee et al., 2007), in part due to the large number of proteins in our interactome that have multiple predicted subcellular localizations while in the Arabidopsis approach single subcellular localization was assigned to each protein through winner-takes-all strategy. We were unable to use the same strategy since Plant$\mathrm{mPloc}$ is our only source for subcellular localization determination. In our analysis, one predicted interaction is scored as belonging to several compartment pairs thereby increasing the number of interologs in these compartment pairs, which could cause no depletion. Additionally, the accuracy of the Plant-mPloc prediction must also be considered. Nevertheless, a clear tendency that interologs are more likely enriched in the compartment pairs paired with same or adjacent compartment can be found from our analysis.

\section{Rice interactome visualization}

A predicted interactome is usually stored in a table with columns and rows. However, in essence it is a network which consists of nodes and edges, which is better visualized as a graph. The Rice Interactions Viewer (RIV; Figure 6), which was developed based on the infrastructure of Arabidopsis Interactions Viewer (AIV), is such a web-based interactome network visualization tool that allows users to perform customized query and analysis (Geisler-Lee et al. 2007). Besides the replacement of the AIV data set with the rice interolog data set described here and the amendment of GUI text, some redundant code was also corrected for the purpose of functional expansion. One major improvement on RIV is the integration of CytoscapeWeb (Lopes et al., 2010; http:// cytoscapeweb.cytoscape.org/). By using the graph definition language GraphML, the color of each protein (node) is painted differently based on its predicted subcellular localization (Figure 6a). Alternatively, nodes can be colored according to its expression level in a specific tissue/condition (Figure 6b), based on rice gene expression data sets stored in the Bio-Array Resource for Plant Biology (Toufighi et al., 2005). For each edge which connects a pair of proteins, the RIV is able to show different color based on the coexpression value (PCC). As well the relative thickness corresponds to its Confidence Value (CV). Moreover, a simple mouse click on each protein (node) is able to show its ID, annotation and predicted subcellular localization.

\section{Conclusions}

\section{How to use the predicted interactome}

A rice interactome has been predicted based on conservation of protein interactions across species over the course of evolution. Each interaction has been assigned a confidence level (CV) as an internal quality control. Assignments for subcellular localization and coexpression can be used to further validate the predicted interaction. Coexpression analysis has shown that interacting proteins tend to have similar expression profiles, and tend to be localized to the same or adjacent compartment. By inputting rice proteins of interest, the Rice Interactions Viewer (Figure 6) can be used to display the results of a query of our predicted interactome in a tablular format and CytoscapeWeb network graph format. In table format output, for the interologs confirmed by published literature, users can link to the paper by clicking the assigned PubMedID. Users can also sort any column easily by clicking the column header. This function is very useful for users who want to check the highest coexpression or confidence level in the output of 


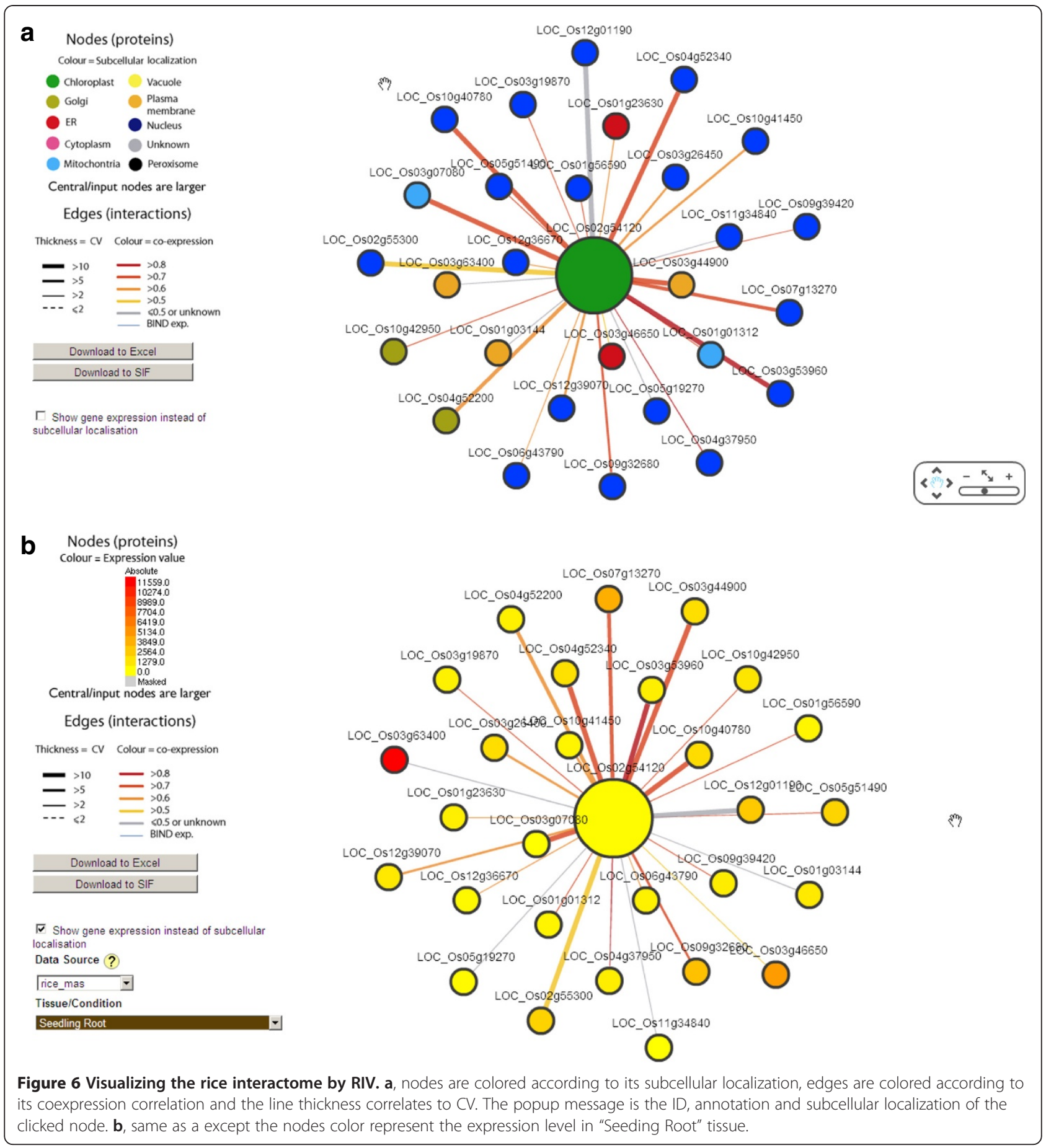

interologs. This predicted interactome is not without limits however, and some caution should be observed before concluding on any positive and especially negative results (interactions expected but not found). Firstly, the interactions predicted are based on orthology between rice and other eukaroytes with experimentally-determined interactomes. Other than about 4000 interactions in Arabidopsis, no other plant has a large number of experimentally-determined interactions to draw from. With the possible exception of yeast (S. cerevisiae), none of the experimental data sets from reference organisms are complete. The interactions contained in this data set are of evolutionarily conserved proteins and pathways. However as the experimental data are based on high throughput studies, the predicted rice interactome does not represent only well studied interactions like the 
proteosome, ribosome and base transcriptional machinery: there are still some surprising connections and a number of unknown proteins within the data set as well.

Note added in revision: we expanded the number of predictions by incorporating Braun et al.'s Arabidopsis Interactome data set (Arabidopsis Interactome Mapping 2011) and also from an Arabidopsis G-protein network (Klopffleisch et al. 2011). A predicted rice interactome by $\mathrm{Gu}$ et al. (2011) was published while this manuscript was under review. While $\mathrm{Gu}$ et al. used similar methodologies, our study had significantly more reference species and thus has improved resolution of conserved interactions.

\section{Methods}

\section{Prediction of orthologs}

Peptide sequences from rice (Oryza sativa subsp. japonica), Arabidopsis thaliana, Homo sapiens, Mus musculus, Rattus norvegicus, Drosophila melanogaster, Caenorhabditis elegans, Saccharomyces cerevisiae, Schizosaccharomyces pombe, Escherichia coli, Bacillus subtilis, Helicobacter pylori were retrieved from TAIR (www.arabidopsis.org; Swarbreck et al., 2008), ENSEMBL (www.ensembl.org/ index.html; Flicek et al., 2010) for the prediction of orthologs using INPARANOID 3.0 (inparanoid.cgb.ki.se, O'Brien et al. 2005) at BLOSUM 80 for rice- $A$. thaliana, BLOSUM 80 and 62 for rice- H. sapiens, M. musculus, $R$. norvegicus and S. cerevisiae; BLOSUM 62 for D. melanogaster, C. elegans, Sch. pombe and BLOSUM 45 for E. coli, B. subtilis and $H$. pylori. Only ortholog pairs that had a score of $100 \%$ confidence (From INPARANOID 3.0) were retrieved for the prediction of rice interactome.

\footnotetext{
Mapping of rice orthologs to reference interactomes Interactome databases were obtained from BioGRID (version 47; www.thebiogrid.org; Breitkreutz et al., 2008), DIP (October 2008 release; dip.doe.mbi.ucla.edu; Salwinski et al., 2004); and IntAct (downloaded December 5, 2009, http://www.ebi.ac.uk/intact; Aranda et al., 2010), respectively. Rice orthologs were mapped onto interactome data using a cross-reference tables assembled from identifiers retrieved from ENSEMBL, The Arabidopsis Information Resource (TAIR; Swarbreck et al., 2008) and The Institute for Genomic Research (JCVI; www.jcvi.org; formerly TIGR). A predicted interactome was recorded in an Excel spreadsheet when both interacting proteins in a reference species had orthologs in rice. Duplicates of the same interactions were removed from different interactome data sets and from different reference species to generate unique interacting protein pairs.
}

\section{Calculation of the confidence value (CV) for experimental support}

The confidence value (CV) for experimental support of a predicted interaction was calculated using the following formula, $\mathrm{CV}=\mathrm{N} \times \mathrm{E} \times \mathrm{S}$; where $\mathrm{N}$ is the total number of data sets in which the interaction was recorded, $E$ is the number of different experimental methods from which an interaction was inferred, and $\mathrm{S}$ is the number of reference species from which the same interaction was recorded. The higher the $\mathrm{CV}$, the more likely an interaction is to be conserved in multiple species and to be more convincing as it is supported by different experimental methods. The interactions were classified according to their $\mathrm{CV}$ to three groups: low confidence $(\mathrm{CV}=1)$, medium confidence $(\mathrm{CV}=2$ to 10$)$ and high confidence $(\mathrm{CV}>10)$.

\section{Mapping of rice orthologs to Gene Ontology (GO)}

Gene Ontology (GO) terms for rice were retrieved from the GO site (Revision 1.52 validated 30 August 2008; http://geneontology.org). The top 7 enriched GOSlim terms for molecular functions (MF) and cellular component $(\mathrm{CC})$, respectively; were used as node features in the visualization of protein network using Cytoscape 2.6 (Lopes et al., 2010; chianti.ucsd.edu).

\section{Mapping of rice orthologs to PFAM domains}

The PFAM data of rice proteins were retrieved from Rice Genome Annotation Project from Michigan State University, USA (rice.plantbiology.msu.edu/). Some of the proteins involved in the interactome may possess more than one PFAM domain, thus all possible pairs of PFAM domains of the interacting protein pairs were queried using MySql (Additional file 4: Table S4). The fold enrichment $(F)$ was calculated as the observed number of PFAM pairs in the rice predicted interactions $(\mathrm{O})$ divided by the expected number of PFAM pairs in the rice predicted interactions $(\mathrm{E})$, where $\mathrm{E}$ is the total number of PFAM pairs in the rice predicted interactions $\mathrm{x}$ (frequency of PFAM domain of protein A $x$ frequency of PFAM domain of protein $B$ found in the total number of unique interacting proteins).

\section{Subcellular localization prediction and enrichment analysis}

The subcellular localization of 67,393 rice proteins was predicted by Plant-mPLoc which covers 12 distinct cellular compartments: chloroplast, nucleus, cytoplasm, mitochondrion, cell wall, cell membrane, peroxisome, endoplasm reticulum, golgi apparatus, vacuole, plastid and extracellular. Due to the existence of splice variants and the nature of protein sorting and trafficking, 8,970 proteins have multiple predicted subcellular localizations. To assess the statistical significance of the enrichment of different subcellular 
compartment pairs in the predicted rice interactome, we used a randomization algorithm. The $\mathrm{P}$ value for the observed numbers of interologs is calculated using a Poisson

$$
P\left(n_{a b}\right)= \begin{cases}\sum_{j=0}^{n_{a b}} \overline{n_{a b}} j \frac{e(-n \bar{a} b)}{j !} & n_{a b}<n_{\overline{a b}}(\text { deplation }) \\ \sum_{j=n_{a b}}^{\infty} \overline{n_{a b}} j \frac{e(-n \bar{a} b)}{j !} & n_{a b}<\overline{n_{a b}}(\text { enrichmemt })\end{cases}
$$

distribution:

Here $n_{a b}$ is the observed number of interologs in our predict interactome where one protein is in compartment $\boldsymbol{a}$ and the other in $\boldsymbol{b} . n_{a b}$ is given by:

$$
n_{a b}=\sum_{j} \sum_{i<j}\left(C_{i a} C_{j b} \text { OR } C_{i b} C_{j a}\right) e i j
$$

- $C_{i a}=1$ or 0 , whether protein $i$ is in compartment $a$ or not

- $C_{i b}=1$ or 0 , whether protein $i$ is in compartment $b$ or not

- $e_{i j}=1$ or 0 , whether protein $i$ and protein $j$ interacts or not

Here $n_{a b}$ is the expected number of interologs with one protein in location $\boldsymbol{a}$ and the other in location $\boldsymbol{b}$ for the ensemble of random protein networks that maintain the following properties as the predicted network: the annotation of proteins in their subcellular localization, the degree $(k k)$ of each protein (the number of proteins that interact with it), and the total number of interacting pairs $(E E), n_{a b}$ is given by

$$
\begin{gathered}
\overline{n_{a b}}=\sum_{j} \sum_{i<j}\left(C_{i a} C_{j b} \text { OR } C_{i b} C_{j a}\right) \overline{e_{i j}} \\
\overline{e_{i j}}=\frac{k_{i} k_{j}}{\left(2 E+k_{1} k_{j}\right)} \text { and } E=\frac{1}{2} \sum_{i} k_{i}
\end{gathered}
$$

- $C_{i a}=1$ or 0 , whether protein $i$ is in compartment $a$ or not

- $C_{i b}=1$ or 0 , whether protein $i$ is in compartment $b$ or not

- $k_{i} k_{j}=$ the node degree of protein(node) $i, j$

- $e_{\mathrm{ij}}=$ the probability of protein(node) $\mathrm{i}$ and protein (node) $\mathrm{j}$ interacts

- $E=$ total number of interactions(edges)

The $\mathrm{P}$ values are finally subject to a multiple-test correction $\boldsymbol{P}$ (multi) $=1-(1-\boldsymbol{P})^{\mathrm{m}}$ where for enrichment $\boldsymbol{m}$ equals the number of $a b$ pairs with at least one observed interolog and for depletion $m$ equals the number of $a b$ pairs possible in the ensemble of random networks.

\section{Coexpression analysis}

Coexpression between interacting proteins was determined using the Pearson correlation coefficient

$$
r=\frac{1}{N} \sum_{i=1 . N}\left(\frac{X_{i}-\bar{X}}{\sigma_{x}}\right)\left(\frac{Y_{i}-\bar{Y}}{\sigma_{y}}\right)
$$

Where $\mathrm{N}$ is the number of expression samples, $\mathrm{X}$ is the expression level for gene $\mathrm{X}$ in $i^{\text {th }}$ sample, and $\mathrm{Y}$ is the expression level for gene $\mathrm{Y}$ in the $i^{\text {th }}$ sample $(-1 \leq \boldsymbol{r} \leq 1)$.

High positive $\boldsymbol{r}$ values indicate a correlation of expression patterns, while low negative $r$ values indicated anticorrelation. A large multi data set compendium consisting of 165 diverse data sets (see Additional file 3: Table S3) was used to generate an accurate representation of gene expression. To determine whether the interolog coexpression distribution was enriched in pairs that exhibited high correlation coefficients, we performed a two-sample Kolomogorov-Smirnov test on the interolog and random distributions. Random distributions were generated by randomly generating protein pairs within the protein collection of our predicted interactome, from any of proteins in whole rice genome, or from any protein in whole rice genome such that the topology of this random set matched that of our predicted interactome in terms of degree distribution.

\section{Additional files}

Additional file 1: Table S1. The rice predicted interactome.

Additional file 2: Table S2. PFAM domains for interacting pairs.

Additional file 3: Table S3. List of GSM identifiers of microarray expression data.

Additional file 4: Table S4. Experimental verification of predicted interactions.

\section{Authors Contributions}

$\mathrm{CLH}$ and MG performed the ortholog and interolog calculations to predict interactions, and computed the confidence value, GO and domain analysis. NP, HBC and YW calculated co-expression, sub-cellular localization prediction, and statistical analysis. NP is the webmaster and created the Rice Interactions Viewer. All authors contributed to the overall development and direction of this project through strong interaction between project members. All authors read and approved the final manuscript.

\section{Acknowledgements}

C.-L. Ho would like to acknowledge the funding of Fulbright Visiting Scholar Grant from the Department of State of U.S.A. and Universiti Putra Malaysia; and the facilities provided by the Department of Plant Biology, Southern Illinois University Carbondale for her research.

\section{Author details}

${ }^{1}$ Faculty of Biotechnology and Biomolecular Sciences, Universiti Putra Malaysia, 43400 UPM-Serdang, Selangor, Malaysia. ${ }^{2}$ Department of Cell \& Systems Biology/Centre for the Analysis of Genome Evolution and Function, 
University of Toronto, Toronto, ON M5S 3B2, Canada. ${ }^{3}$ Department of Automation, Shanghai Jiao Tong University, No.800 Dongchuan RoadShanghai 200240, China. ${ }^{4}$ Department of Cell \& Systems Biology/Centre for the Analysis of Genome Evolution and Function, University of Toronto, Toronto, ON M5S 3B2, Canada. ${ }^{5}$ Department of Plant Biology, Southern Illinois University Carbondale, 1125 Lincoln Ave., Life Science II, Carbondale, IL 62901-6509, USA.

Received: 7 June 2012 Accepted: 13 June 2012

Published: 2 July 2012

\section{References}

Alon U (2007) Network motifs: theory and experimental approaches. Nat Rev Genet 8:450-461

Arabidopsis Interactome Mapping Consortium (2011) Evidence for network evolution in an Arabidopsis interactome map. Science 333:601-607

Aranda B, Achuthan P, Alam-Faruque Y, Armean I, Bridge A, Derow C, Feuermann M, Ghanbarian AT, Kerrien S, Khadake J, Kerssemakers J, Leroy C, Menden M, Michaut M, Montecchi-Palazzi L, Neuhauser SN, Orchard S, Perreau V, Roechert B, van Eijk K, Hermjakob H (2010) The IntAct molecular interaction database in 2010. Nucleic Acids Res 38:D525-D531

Arora R, Agarwal P, Ray S, Singh A, Singh V, Tyagi A, Kapoor S (2007) MADS-box gene family in rice: genome-wide identification, organization and expression profiling during reproductive development and stress. BMC Genomics 8:242

Bader GD, Donaldson I, Wolting C, Ouellette BFF, Pawson T, Hogue CWV (2001) BIND - The biomolecular interaction network database. Nucleic Acids Res 29:242-245

Arabidopsis Interactome Mapping C (2011) Evidence for network evolution in an Arabidopsis interactome map. Science 333:601-607

Breitkreutz BJ, Stark C, Reguly T, Boucher L, Breitkreutz A, Livstone M, Oughtred R, Lackner DH, Bahler J, Wood V, Dolinski K, Tyers M (2008) The BioGRID interaction database: 2008 update. Nucleic Acids Res 36:D637-D640

Ceol A, Chatr Aryamontri A, Licata L, Peluso D, Briganti L, Perfetto L, Castagnoli L, Cesareni G (2009) MINT, the molecular interaction database. Nucleic Acids Res 38:D532-D539

Chou KC, Shen HB (2010) Plant-mPLoc: a top-down strategy to augment the power for predicting plant protein subcellular localization. PLoS One 5:e9931. doi:10.1371

Cler E, Papai G, Schultz P, Davidson I (2009) Recent advances in understanding the structure and function of general transcription factor TFIID. Cell Mol Life Sci 66:2123-2134

Cooper B, Clarke JD, Budworth P, Kreps J, Hutchison D, Park S, Guimil S, Dunn M, Luginbuhl P, Ellero C, Goff SA, Glazebrook J (2003a) A network of rice genes associated with stress response and seed development. Proc Natl Acad Sci USA 100:4945-4950

Cooper B, Hutchison D, Park S, Guimil S, Luginbuhl P, Ellero C, Goff SA, Glazebrook J (2003b) Identification of rice (Oryza sativa) proteins linked to the cyclin-mediated regulation of the cell cycle. Plant Mol Biol 53:273-279

de Folter S, Immink RGH, Kieffer M, Parenicova L, Henz SR, Weigel D, Busscher M, Kooiker M, Colombo L, Kater MM, Davies B, Angenent GC (2005) Comprehensive interaction map of the Arabidopsis MADS box transcription factors. Plant Cell 17:1424-1433

Ding XD, Richter T, Chen M, Fujii H, Seo YS, Xie MT, Zheng XW, Kanrar S, Stevenson RA, Dardick C, Li Y, Jiang H, Zhang Y, Yu FH, Bartley LE, Chern M, Bart R, Chen XH, Zhu LH, Farmerie WG, Gribskov M, Zhu JK, Fromm ME, Ronald PC, Song WY (2009) A Rice kinase-protein interaction map. Plant Physiol 149:1478-1492

Donoso I, Munoz-Centeno MC, Sanchez-Duran MA, Flores A, Daga RR, Guevara CM, Bejarano ER (2005) Mpg1, a fission yeast protein required for proper septum structure, is involved in cell cycle progression through cell-size checkpoint. Mol Genet Genomics 274:155-167

Dortay H, Gruhn N, Pfeifer A, Schwerdtner M, Schmuälling T, Heyl A (2008) Toward an Interaction Map of the two-component signaling pathway of Arabidopsis thaliana. J Proteome Res 7:3649-3660

Flicek P, Aken BL, Ballester B, Beal K, Bragin E, Brent S, Chen Y, Clapham P, Coates G, Fairley S, Fitzgerald S, Fernandez-Banet J, Gordon L, Graf S, Haider S, Hammond M, Howe K, Jenkinson A, Johnson N, Kahari A, Keefe D, Keenan S, Kinsella R, Kokocinski F, Koscielny G, Kulesha E, Lawson D, Longden I, Massingham T, McLaren W, Megy K, Overduin B, Pritchard B, Rios D, Ruffier M, Schuster M, Slater G, Smedley D, Spudich G, Tang YA, Trevanion S, Vilella A, Vogel J, White S, Wilder SP, Zadissa A, Birney E, Cunningham F, Dunham I, Durbin R, Fernandez-
Suarez XM, Herrero J, Hubbard TJP, Parker A, Proctor G, Smith J, Searle SMJ (2010) Ensembl's 10th year. Nucleic Acids Res 38:D557-D562

Gandhi TKB, Zhong J, Mathivanan S, Karthick L, Chandrika KN, Mohan SS, Sharma S, Pinkert S, Nagaraju S, Periaswamy B, Mishra G, Nandakumar K, Shen BY, Deshpande N, Nayak R, Sarker M, Boeke JD, Parmigiani G, Schultz J, Bader JS, Pandey A (2006) Analysis of the human protein interactome and comparison with yeast, worm and fly interaction datasets. Nat Genet 38:285-293

Geisler-Lee J, O'Toole N, Ammar R, Provart NJ, Millar AH, Geisler M (2007) A predicted interactome for Arabidopsis. Plant Physiol 145:317-329

Giot L, Bader JS, Brouwer C, Chaudhuri A, Kuang B, Li Y, Hao YL, Ooi CE, Godwin B, Vitols E, Vijayadamodar G, Pochart P, Machineni H, Welsh M, Kong Y, Zerhusen B, Malcolm R, Varrone Z, Collis A, Minto M, Burgess S, McDaniel L, Stimpson E, Spriggs F, Williams J, Neurath K, loime N, Agee M, Voss E, Furtak K, Renzulli R, Aanensen N, Carrolla S, Bickelhaupt E, Lazovatsky Y, DaSilva A, Zhong J, Stanyon CA, Finley RL, White KP, Braverman M, Jarvie T, Gold S, Leach M, Knight J, Shimkets RA, McKenna MP, Chant J, Rothberg JM (2003) A protein interaction map of Drosophila melanogaster. Science 302:1727-1736

Goldberg AL, Rock KL (1992) Proteolysis, proteasomes and antigen presentation. Nature 357:375-379

Gorynia S, Matias PM, Goncalves S, Coelho R, Lopes G, Thomaz M, Huber M, Haendler B, Donner P, Carrondo MA (2006) Expression, purification, crystallization and preliminary X-ray analysis of the human RuvB-like protein RuvBL1. Acta Crystallogr Sect F Struct Biol Cryst Commun 62:61-66

Gregis V, Sessa A, Colombo L, Kater MM (2006) AGL24, SHORT VEGETATIVE PHASE, and APETALA1 redundantly control AGAMOUS during early stages of flower development in Arabidopsis. Plant Cell 18:1373-1382

Gu Y, Deng Z, Paredez AR, DeBolt S, Wang ZH, Somerville C (2008) Prefoldin 6 is required for normal microtubule dynamics and organization in Arabidopsis. PNAS 105(46):18064-18069

Gu H, Zhu P, Jiao Y, Meng Y, Chen M (2011) PRIN: a predicted rice interactome network. BMC Bioinformatics 12:161

Hilt W, Wolf DH (1996) Proteasomes: destruction as a programme. Trends Biochem Sci 21:96-102

Klopffleisch K, Phan N, Augustin K, Bayne RS, Booker KS, Botella JR, Carpita NC, Carr T, Chen J-G, Cooke TR, Frick-Cheng A, Friedman EJ, Fulk B, Hahn MG, Jiang K, Jorda L, Kruppe L, Liu C, Lorek J, McCann MC, Molina A, Moriyama EN, Mukhtar MS, Mudgil Y, Pattathil S, Schwarz J, Seta S, Tan M, Temp U, Trusov Y, Urano D, Welter B, Yang J, Panstruga R, Uhrig JF, Jones AM (2011) Arabidopsis $\mathrm{G}$-protein interactome reveals connections to cell wall carbohydrates and morphogenesis. Mol Syst Biol 7(Article number):532

Li SM, Armstrong CM, Bertin N, Ge H, Milstein S, Boxem M, Vidalain PO, Han JDJ, Chesneau A, Hao T, Goldberg DS, Li N, Martinez M, Rual JF, Lamesch P, Xu L, Tewari M, Wong SL, Zhang LV, Berriz GF, Jacotot L, Vaglio P, Reboul J, Hirozane-Kishikawa T, Li QR, Gabel HW, Elewa A, Baumgartner B, Rose DJ, Yu HY, Bosak S, Sequerra R, Fraser A, Mango SE, Saxton WM, Strome S, van den Heuvel S, Piano F, Vandenhaute J, Sardet C, Gerstein M, Doucette-Stamm L, Gunsalus KC, Harper JW, Cusick ME, Roth FP, Hill DE, Vidal M (2004) A map of the interactome network of the metazoan C-elegans. Science 303:540-543

Liu JX, Howell SH (2010) bZIP28 and NF-Y Transcription factors are activated by ER stress and assemble into a transcriptional complex to regulate stress response genes in Arabidopsis. Plant Cell 22:782-796

Lopes CT, Franz M, Kazi F, Donaldson SL, Morris Q, Bader GD (2010) Cytoscape Web: an interactive web-based network browser. Bioinformatics 26(18):2347-2348

Miller JP, Lo RS, Ben-Hur A, Desmarais C, Stagljar I, Noble WS, Fields S (2005) Large-scale identification of yeast integral membrane protein interactions. Proc Natl Acad Sci USA 102:12123-12128

Narayanan M, Vetta A, Schadt EE, Zhu J (2010) Simultaneous clustering of multiple gene expression and physical interaction datasets. PLoS Comput Biol 6:4

O'Brien KP, Remm M, Sonnhammer ELL (2005) Inparanoid: a comprehensive database of eukaryotic orthologs. Nucleic Acids Res 33:D476-D480

Pagel P, Kovac S, Oesterheld M, Brauner B, Dunger-Kaltenbach I, Frishman G, Montrone C, Mark P, Stumpflen V, Mewes HW, Ruepp A, Frishman D (2005) The MIPS mammalian protein-protein interaction database. Bioinformatics $21: 832-834$

Pelaz S, Ditta GS, Baumann E, Wisman E, Yanofsky MF (2000) B and C floral organ identity functions require SEPALLATA MADS-box genes. Nature 405:200-203

Rivett AJ (1993) Proteasomes - Multicatalytic Proteinase Complexes. Biochem J 291:1-10

Rounsley SD, Ditta GS, Yanofsky MF (1995) Diverse roles for mads box genes in Arabidopsis development. Plant Cell 7:1259-1269 
Rual JF, Venkatesan K, Hao T, Hirozane-Kishikawa T, Dricot A, Li N, Berriz GF, Gibbons FD, Dreze M, Ayivi-Guedehoussou N, Klitgord N, Simon C, Boxem M, Milstein S, Rosenberg J, Goldberg DS, Zhang LV, Wong SL, Franklin G, Li SM, Albala JS, Lim JH, Fraughton C, Llamosas E, Cevik S, Bex C, Lamesch P, Sikorski RS, Vandenhaute J, Zoghbi HY, Smolyar A, Bosak S, Sequerra R, Doucette-Stamm L, Cusick ME, Hill DE, Roth FP, Vidal M (2005) Towards a proteome-scale map of the human protein-protein interaction network. Nature 437:1173-1178

Salwinski L, Miller CS, Smith AJ, Pettit FK, Bowie JU, Eisenberg D (2004) The database of interacting proteins: 2004 update. Nucleic Acids Res 32:D449-D451

Springer PS, Holding DR, Groover A, Yordan C, Martienssen RA (2000) The essential Mcm7 protein PROLIFERA is localized to the nucleus of dividing cells during the $\mathrm{G}(1)$ phase and is required maternally for early Arabidopsis development. Development 127:1815-1822

Sridhar W, Surendrarao A, Liu ZC (2006) APETALA1 and SEPALLATA3 interact with SEUSS to mediate transcription repression during flower development (vol 133, pg 3159, 2001). Development 133:3496

Swarbreck D, Wilks C, Lamesch P, Berardini TZ, Garcia-Hernandez M, Foerster H, Li D, Meyer T, Muller R, Ploetz L, Radenbaugh A, Singh S, Swing V, Tissier C, Zhang P, Huala E (2008) The Arabidopsis information resource (TAIR): gene structure and function annotation. Nucleic Acids Res 36:D1009-D1014

Theissen G, Melzer R (2007) Molecular mechanisms underlying origin and diversification of the angiosperm flower. Ann Bot 100:603-619

Toufighi K, Brady SM, Austin R, Ly E, Provart NJ (2005) The botany array resource: e-Northerns, expression angling, and promoter analyses. Plant J 43:153-163

Uetz P, Giot L, Cagney G, Mansfield TA, Judson RS, Knight JR, Lockshon D, Narayan V, Srinivasan M, Pochart P, Qureshi-Emili A, Li Y, Godwin B, Conover D, Kalbfleisch T, Vijayadamodar G, Yang MJ, Johnston M, Fields S, Rothberg JM (2000) A comprehensive analysis of protein-protein interactions in Saccharomyces cerevisiae. Nature 403:623-627

Wiles AM, Doderer M, Ruan JH, Gu TT, Ravi D, Blackman B, Bishop AJR (2010) Building and analyzing protein interactome networks by cross-species comparisons. BMC Syst Biol 4:36. doi:10.1186/1752-0509-4-36

Wilk S (1993) Proteasomes - multicatalytic proteinase complexes. Enzyme protein 47:187-188

Yung MH, Schaffer R, Putterill J (1999) Identification of genes expressed during early Arabidopsis carpel development by mRNA differential display: characterisation of ATCEL2, a novel endo-1,4-beta-D-glucanase gene. Plant J 17:203-208

Zanzoni A, Montecchi-Palazzi L, Quondam M, Ausiello G, Helmer-Citterich M, Cesareni G (2002) MINT: a molecular interaction database. FEBS Lett 513:135-140

Zaslaver A, Mayo AE, Rosenberg R, Bashkin P, Sberro H, Tsalyuk M, Surette MG, Alon U (2004) Just-in-time transcription program in metabolic pathways. Nat Genet 36:486-491

doi:10.1186/1939-8433-5-15

Cite this article as: Ho et al: A predicted protein interactome for rice. Rice 2012 5:15.

\section{Submit your manuscript to a SpringerOpen ${ }^{\circ}$ journal and benefit from:}

- Convenient online submission

- Rigorous peer review

- Immediate publication on acceptance

- Open access: articles freely available online

- High visibility within the field

- Retaining the copyright to your article

Submit your next manuscript at $\gg$ springeropen.com 